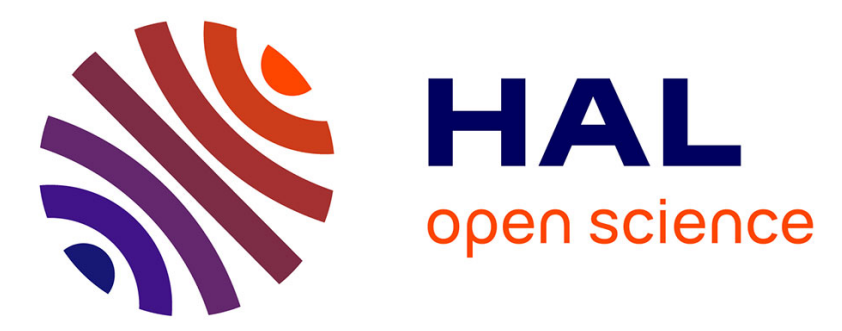

\title{
Accurate artificial boundary conditions for the semi-discretized linear Schrödinger and heat equations on rectangular domains
}

\author{
Songsong Ji, Yibo Yang, Gang Pang, Xavier Antoine
}

\section{To cite this version:}

Songsong Ji, Yibo Yang, Gang Pang, Xavier Antoine. Accurate artificial boundary conditions for the semi-discretized linear Schrödinger and heat equations on rectangular domains. Computer Physics Communications, 2018, 222, pp.84-93. 10.1016/j.cpc.2017.09.019 . hal-01649707

\section{HAL Id: hal-01649707 https://hal.science/hal-01649707}

Submitted on 27 Nov 2017

HAL is a multi-disciplinary open access archive for the deposit and dissemination of scientific research documents, whether they are published or not. The documents may come from teaching and research institutions in France or abroad, or from public or private research centers.
L'archive ouverte pluridisciplinaire HAL, est destinée au dépôt et à la diffusion de documents scientifiques de niveau recherche, publiés ou non, émanant des établissements d'enseignement et de recherche français ou étrangers, des laboratoires publics ou privés. 


\title{
Accurate artificial boundary conditions for the semi-discretized linear Schrödinger and heat equations on rectangular domains
}

\author{
Songsong JI ${ }^{\mathrm{a}}$, Yibo YANG $^{\mathrm{b}}$, Gang PANG ${ }^{\mathrm{c}, *}$, Xavier ANTOINE ${ }^{\mathrm{d}}$ \\ ${ }^{a}$ College of Engineering, Peking University, Beijing, P. R. China \\ ${ }^{b}$ Department of Statistics, Chicago University, Chicago, USA \\ ${ }^{c}$ Institute of Applied Physics and Computational Mathematics, Beijing, 100088, P.R. China \\ ${ }^{d}$ Université de Lorraine, Institut Elie Cartan de Lorraine, SPHINX team, Inria Nancy-Grand Est, F-54506 Vandoeuvre-lès-Nancy Cedex, France
}

\begin{abstract}
The aim of this paper is to design some accurate artificial boundary conditions for the semi-discretized linear Schrödinger and heat equations in rectangular domains. The Laplace transform in time and discrete Fourier transform in space are applied to get the Green's functions of the semi-discretized equations in unbounded domains with single-source. An algorithm is given to compute these Green's functions accurately through some recurrence relations. Furthermore, the finite-difference method is used to discretize the reduced problem with accurate boundary conditions. Numerical simulations are presented to illustrate the accuracy of our method in the case of the linear Schrödinger and heat equations. It is shown that the reflection at the corners is correctly eliminated.
\end{abstract}

Keywords: Schrödinger equation, heat equation, semi-discretization, rectangular boundary, artificial boundary conditions, Green's function

2010 MSC: 81Q05, 35Q41, 65M06,

\section{Introduction}

The Schrödinger and heat equations in infinite domains are standard models with many interesting applications in computational physics and engineering. The Schrödinger equation is one of the fundamental equations arising in quantum mechanics [1], but has also many other important applications including electromagnetic/acoustic wave propagation [2] through the so-called parabolic equation, plasma physics [3], seismic migration [4] or optical fibers with the Fresnel equation [5]. The heat equation defined on an unbounded spatial domain arises as a model for fluid dynamics [6] and also for financial applications [7]. The numerical solution and analysis of these linear PDEs on unbounded domains has so far received a great attention [8]. To get a reliable computational method, a standard approach is to truncate the infinite domain around the region of interest and to implement an artificial boundary condition on the fictitious boundary (see e.g. $[9,10]$ for Schrödinger-like equations). Alternative strategies include the Perfectly Matched Layer (PML) (and also Complex Absorbing Scaling (CAS)) [9, 10].

There are rather extensive studies on artificial boundary conditions for the one-dimensional case. For instance and without being exhaustive, Fevens and Jiang [11] constructed some local artificial boundary conditions. In [12, 13], the authors derive a stable quadrature rule for approximating the transparent operator at the boundary and localizations through Padé approximations of the exact operator. Wu and Zhang [14] constructed some high-order local absorbing boundary conditions, and avoided high-order derivatives by using auxiliary variables on the fictitious boundary. For the theoretical analysis, Arnold et al. [15] proposed an efficient way to treat the Schrödinger equation, and proved the stability of the resulting initial boundary-value scheme. For the heat equation, Greengard and Lin [16] proposed an algorithm based on the evolution of the continuous spectrum for the heat equation on unbounded domains. Wu

\footnotetext{
${ }^{*}$ Corresponding author

Email addresses: songsong.0211@163.com (Songsong JI), ybyang@uchicago.edu (Yibo YANG), 1517191281@qq.com (Gang PANG), xavier. antoine@univ-lorraine.fr (Xavier ANTOINE)
} 
and Sun [17] proved the unconditional stability for the heat equation when a well-designed finite-difference scheme is used.

For the two-dimensional case, Baskakov and Popov [18] obtained the exact transparent boundary conditions for the Schrödinger equation in a half-plane. Han and Huang $[19,20]$ constructed an exact artificial boundary condition to reduce the original heat equation to an initial boundary-value problem on a finite computational domain. They also derived the exact artificial boundary condition for the two-dimensional Schrödinger equation by expressing the solution with Hankel's functions, implemented with a cut-off. In [21], Arnold et al. proposed some discretized transparent boundary conditions for the time-dependent Schrödinger equation on a circular computational domain, and illustrated the accuracy, stability, and efficiency of the proposed method. Zhang et al. [22] designed high-order artificial boundary conditions for the two-dimensional Schrödinger equation on a circular boundary by rationally approximating the kernel functions. For more general cases, Antoine et al. [23] constructed and studied a Crank-Nicolson-type discretization for some increasing order approximations of the non-reflecting boundary condition. They also proposed some absorbing boundary conditions for general nonlinear and two-dimensional Schrödinger equations with an exterior potential (see [24, 25, 26]). Their approach relies on pseudo-differential operators to construct truncated boundary conditions, which can be proved to be stable. Most of these boundary conditions are proposed on a circular or smooth boundary and need a truncation rule in the final implementation. We refer to [9] for an extensive survey for the Schrödinger equation, and to [10] for a recent overview for absorbing boundary conditions and PMLs for the Schrödinger, Klein-Gordon and Dirac equations.

Considering a rectangular/square computational domain is natural in practice, but deriving an associated accurate artificial boundary condition is non trivial most particularly because of the presence of the singular corner points. Until now, there are only a few results for building accurate boundary conditions for the Schrödinger, wave and heat equations on a rectangular computational domain. Some contributions can be found e.g. in the references [8, 27]. To deal with the Schrödinger and heat equations on unbounded domains, we propose first to introduce a second-order spatial discretization of the associated operator on a uniform rectangular grid. Inspired by our previous work in lattice dynamics [28], an efficient algorithm is designed to accurately compute the Green's function of the semi-discretized Schrödinger equation with a single-source. Based on this computation, we are able to design some accurate boundary conditions that eliminate the corner reflection when a rectangular boundary is considered.

The paper is organized as follows. In Section 2, the Green's functions of the semi-discretized equations on unbounded domains with a single-source are derived and an accurate algorithm is designed for computing the Green's functions. They are next used to build the accurate artificial boundary conditions for both the time-dependent Schrödinger and heat equations. In Section 3, the fully discretized schemes are presented for the Schrödinger and heat equations with the accurate artificial boundary conditions set on a rectangular domain. Numerical examples are reported in Section 4 to demonstrate the accuracy of our method. We end this paper (Section 5) with some concluding remarks and perspectives.

\section{Building accurate artificial boundary conditions for the semi-discretized Schrödinger and heat equations}

We first start in subsection 2.1 by the basic ideas to design accurate artificial boundary conditions for the linear Schrödinger equation. The heat equation is treated shortly in subsection 2.2.

\subsection{The case of the linear Schrödinger equation}

To design some exact artificial boundary conditions, let us rewrite the Schrödinger equation as: find $u:=u(\mathbf{x}, t)$ such that

$$
\begin{aligned}
& \left.\left.i \frac{\partial}{\partial t} u=-\left(\frac{\partial^{2}}{\partial x^{2}}+\frac{\partial^{2}}{\partial y^{2}}\right) u, \quad(\mathbf{x}, t) \text { in } \mathbb{R}^{2} \times\right] 0, T\right], \\
& u(\mathbf{x}, t=0)=0, \quad \mathbf{x} \in \Omega^{\mathrm{ext}}=\mathbb{R}^{2} \backslash \Omega^{\mathrm{int}}, \\
& u(\mathbf{x}, \cdot) \rightarrow 0, \quad \text { for }\|\mathbf{x}\| \rightarrow+\infty .
\end{aligned}
$$

The initial data $u_{0}(\mathbf{x})$ is supposed to be compactly supported into the interior square domain $\left.\Omega^{\text {int }}=\right] 0, a\left[^{2}\right.$ (the extension to a rectangular domain is straightforward up to some additional notations), $\Omega^{\text {ext }}$ being the associated exterior domain. 
The maximal time of computation is $T$. The complex number $i$ is defined by $i:=\sqrt{-1}$. Let us remark that adding a purely time-dependent potential can be directly considered by eliminating the potential effect through a gauge change [12].

Let us introduce $\Delta x$ as the uniform mesh size in the $x$ - and $y$-directions to discretize $\Omega^{\text {int }}$ with $(L+1)$ segments in each direction, i.e. $(L+1) \Delta x=a$. After scaling the time by $(\Delta x)^{2}$, the spatial semi-discretization of $(1)$ with a five-points stencil finite difference scheme gives

$$
\begin{aligned}
& \left.\left.i \dot{u}_{m, n}=-\left(u_{m+1, n}+u_{m-1, n}+u_{m, n+1}+u_{m, n-1}-4 u_{m, n}\right), \quad \text { in } \mathbb{Z}^{2} \times\right] 0, T\right], \\
& \left.u_{m, n}\right|_{t=0}=0, \quad \text { in } \quad(m, n) \in \mathbb{Z}^{2} \backslash\{(p, q) / 1 \leq p \leq L, 1 \leq q \leq L\}, \\
& u_{m, n} \rightarrow 0 \text {, when } \quad|m|,|n| \rightarrow+\infty
\end{aligned}
$$

where $u_{m, n}(t)$ approximates $u(m \Delta x, n \Delta x, t)$, and $\dot{u}_{m, n}$ is the time derivative of $u_{m, n}$. The computation of (4)-(6) should be confined within a finite computational domain $\{(m, n) / 1 \leq m \leq L, 1 \leq n \leq L\}$ where the value of $u_{m, n}$ is needed for $m=0, L+1$, and $n=0, L+1$, to complete system (4) through a boundary relation.

Here we derive the Green's function $f_{m, n}(t)$ of the semi-discretized linear Schrödinger equation with single-source. Let us consider the following system

$$
\begin{aligned}
& \left.\left.i \dot{f}_{m, n}=-\left(f_{m+1, n}+f_{m-1, n}+f_{m, n+1}+f_{m, n-1}-4 f_{m, n}\right), \quad \text { in } \mathbb{Z}^{2} \times\right] 0, T\right], \\
& \left.f_{m, n}\right|_{t=0}=0, \quad \text { in } \mathbb{Z}^{2} \backslash\{(0,0)\}, \\
& f_{m, n} \rightarrow 0, \quad \text { when } \quad|m|,|n| \rightarrow+\infty,
\end{aligned}
$$

where $f_{0,0}(t)=\delta(t)$ is the Dirac distribution, and let us compute the related discrete Green's function. By defining the Laplace transform as

$$
\tilde{f}_{m, n}(s)=\int_{0}^{\infty} f_{m, n}(t) e^{-s t} d t
$$

we obtain, for $(m, n) \neq(0,0)$,

$$
A \tilde{f}_{m, n}=\tilde{f}_{m+1, n}+\tilde{f}_{m-1, n}+\tilde{f}_{m, n+1}+\tilde{f}_{m, n-1},
$$

setting $A=4-s i$. By using the discrete Fourier transform

$$
\tilde{F}(x, y)=\sum_{m=-\infty}^{\infty} \sum_{n=-\infty}^{\infty} e^{i m x} e^{i n y} \tilde{f}_{m, n}
$$

together with (10) gives

$$
\tilde{F}(x, y)=\frac{A-4 \tilde{f}_{0,1}}{A-2 \cos (x)-2 \cos (y)} .
$$

By inverse discrete Fourier transform, one gets

$$
\tilde{f}_{m, n}(s)=\frac{\tilde{g}_{m, n}(s)}{\tilde{g}_{0,0}(s)}=\frac{\frac{1}{4 \pi^{2}} \int_{0}^{2 \pi} \int_{0}^{2 \pi} \frac{e^{-i m x-i n y}}{A-2 \cos (x)-2 \cos (y)} d x d y}{\frac{1}{4 \pi^{2}} \int_{0}^{2 \pi} \int_{0}^{2 \pi} \frac{1}{A-2 \cos (x)-2 \cos (y)} d x d y}
$$

where one has [29]

$$
\begin{aligned}
& \tilde{g}_{m, n}(s)=\frac{1}{4 \pi^{2}} \int_{0}^{2 \pi} \int_{0}^{2 \pi} \frac{e^{-i m x-i n y}}{A-2 \cos (x)-2 \cos (y)} d x d y \\
& =\frac{1}{(4-s i)^{m+n+1}} \frac{(m+n) !}{m ! n !}{ }_{4} F_{3}\left(\begin{array}{c}
\left.\frac{1}{2}(m+n+1), \frac{1}{2}(m+n+1), \frac{1}{2}(m+n)+1, \frac{1}{2}(m+n)+1 ; \frac{1}{\left(1-\frac{s i}{4}\right)^{2}}\right) . \\
m+1, n+1, m+n+1
\end{array}\right)
\end{aligned}
$$


By inverse Laplace transform, we obtain

$$
g_{m, n}(t)=\frac{(m+n) !}{m ! n !} \frac{4 i \sqrt{\pi}(i t)^{m+n} e^{-4 i t}}{2^{m+n+2} \Gamma\left(\frac{m+n+1}{2}\right) \Gamma\left(\frac{m+n}{2}+1\right)}{ }_{2} F_{3}\left(\begin{array}{c}
\frac{m+n+1}{2}, \frac{m+n}{2}+1 ;-4 t^{2} \\
m+1, n+1, m+n+1
\end{array}\right) .
$$

The function $\Gamma$ is the Gamma special function and the generalized hypergeometric function ${ }_{2} F_{3}$ is

$$
{ }_{2} F_{3}\left(\begin{array}{c}
a_{1}, a_{2} ; z \\
b_{1}, b_{2}, b_{3}
\end{array}\right)=\sum_{k=0}^{\infty} \frac{\left(a_{1}\right)_{k}\left(a_{2}\right)_{k}}{k !\left(b_{1}\right)_{k}\left(b_{2}\right)_{k}\left(b_{3}\right)_{k}} z^{k},
$$

with $(a)_{k}=a(a+1) \ldots(a+k-1)$ for $k \geq 1$ and $(a)_{0}=1$. We display in Fig. 1 the $(L+2) \times(L+2)$ square lattice $(0 \leq m, n \leq L+1)$ arising in (4) for $L=4$.

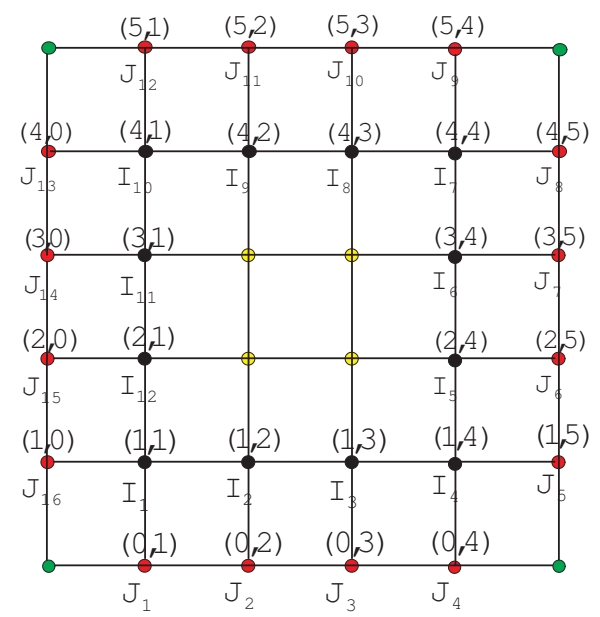

Figure 1: Discrete computational lattice (for $L=4$ ): the red bullets represent the outer layer points, the black bullets correspond to the interior layer points, the yellow bullets are the interior points and the green bullets represent the corner points.

The numerical boundary points are indexed anticlockwise, with the interior layer as $I_{\ell}$ for $\ell=1, \cdots, 4 L-4$. More explicitly, we set: $I_{\ell}=(1, \ell)$ for $1 \leq \ell \leq L, I_{\ell+L-1}=(\ell, L)$ for $1 \leq \ell \leq L, l_{\ell+2 L-2}=(L, L+1-\ell)$ for $1 \leq \ell \leq L$ and $I_{\ell+3 L-3}=(L+1-\ell, 1)$ for $1 \leq \ell \leq L$. The outer layer points are denoted by $J_{k}$ for $k=1, \cdots, 4 L$, with the same orientation. To this end, let $u_{I_{\ell}}(t)$, for $\ell=1, \cdots 4 L-4$, be given. We first decompose them into $(4 L-4)$ independent single-sources $\kappa_{I_{k}}(t)$, namely,

$$
u_{I_{k}}(t)=\sum_{\ell=1}^{4 L-4} f_{I_{k}-I_{\ell}}(t) * \kappa_{I_{\ell}}(t), \quad 1 \leq k \leq 4 L-4 .
$$

Now for $(m, n)$ such that $(m, n) \in \mathbb{Z}^{2} \backslash\{(p, q) / 1 \leq p \leq L, 1 \leq q \leq L\}$, we define the Laplace transform of $u_{m, n}(t)$ as

$$
\tilde{u}_{m, n}(s)=\sum_{\ell=1}^{4 L-4} \frac{\tilde{g}_{(m, n)-I_{\ell}}(s)}{\tilde{g}_{0,0}(s)} \tilde{\kappa}_{I_{\ell}}(s) .
$$

A direct computation shows that, for $|m|>0$ or $|n|>0$,

$$
i s \tilde{g}_{m, n}=-\left(\tilde{g}_{m-1, n}+\tilde{g}_{m+1, n}+\tilde{g}_{m, n-1}+\tilde{g}_{m, n+1}-4 \tilde{g}_{m, n}\right) .
$$

Plugging this expression into (17) yields

$$
\begin{aligned}
& (4-s i) \tilde{u}_{m, n}(s)-\tilde{u}_{m+1, n}(s)-\tilde{u}_{m-1, n}(s)-\tilde{u}_{m, n+1}(s)-\tilde{u}_{m, n-1}(s) \\
& =\sum_{\ell=1}^{4 L-4} \frac{(4-i s) \tilde{g}_{(m, n)-I_{\ell}}-\left(\tilde{g}_{(m-1, n)-I_{\ell}}+\tilde{g}_{(m+1, n)-I_{\ell}}+\tilde{g}_{(m, n-1)-I_{\ell}}+\tilde{g}_{(m, n+1)-I_{\ell}}\right)}{\tilde{g}_{0,0}(s)} \tilde{\kappa}_{I_{\ell}}(s)=0 . \\
& 4
\end{aligned}
$$


It is also easy to check that $u_{m, n}(0)=0$, which leads to

$$
i \dot{u}_{m, n}=-\left(u_{m+1, n}+u_{m-1, n}+u_{m, n+1}+u_{m, n-1}-4 u_{m, n}\right) .
$$

Therefore, $\left(u_{m, n}(t)\right)_{m, n}$ is exactly the solution of the semi-discretized system (4) outside the domain $\{(p, q) / 1 \leq p \leq$ $L, 1 \leq q \leq L\}$. Once the sources $\kappa_{I_{k}}(t)$ are obtained, an exact boundary condition reads as follows

$$
u_{J_{k}}(t)=\sum_{\ell=1}^{4 L-4} f_{J_{k}-I_{\ell}}(t) * \kappa_{I_{\ell}}(t), \quad 1 \leq k \leq 4 L .
$$

Defining $\tilde{\lambda}_{I_{k}}(s)=\frac{\tilde{\kappa}_{I_{k}}(s)}{\tilde{g}_{0,0}(s)}$, for $1 \leq k \leq 4 L-4$, the boundary conditions can be transformed into

$$
u_{I_{k}}(t)=\sum_{\ell=1}^{4 L-4} g_{I_{k}-I_{\ell}}(t) * \lambda_{I_{\ell}}(t), \quad 1 \leq k \leq 4 L-4,
$$

and

$$
u_{J_{k}}(t)=\sum_{\ell=1}^{4 L-4} g_{J_{k}-I_{\ell}}(t) * \lambda_{I_{\ell}}(t), \quad 1 \leq k \leq 4 L .
$$

Combining the boundary conditions (22) and (23) and the interior equation (4), we obtain the reduced semi-discretized problem. The values $g_{m, n}(t)$ are needed in the boundary conditions (22) and (23). For large $m$ and $n$, it is not easy to compute $g_{m, n}(t)$ precisely through hypergeometric functions for large $t$. Consequently, we need to develop an efficient algorithm for computing $g_{m, n}(t)$ accurately. To this end, one easily shows that

$$
\tilde{g}_{m-1, n}-\tilde{g}_{m+1, n}=\frac{1}{2 \pi^{2}} \int_{0}^{2 \pi} \int_{0}^{2 \pi} \frac{\sin (m x) \sin (x) \cos (n y)}{A-2 \cos (x)-2 \cos (y)} d x d y,
$$

leading to

$$
\begin{aligned}
& i \frac{d}{d s}\left(\tilde{g}_{m-1, n}-\tilde{g}_{m+1, n}\right)=\frac{d}{d A}\left(\tilde{g}_{m-1, n}-\tilde{g}_{m+1, n}\right)=-\frac{1}{2 \pi^{2}} \int_{0}^{2 \pi} \int_{0}^{2 \pi} \frac{\sin (m x) \sin (x) \cos (n y)}{(A-2 \cos (x)-2 \cos (y))^{2}} d x d y \\
& =\frac{1}{4 \pi^{2}} \int_{0}^{2 \pi} \int_{0}^{2 \pi} \sin (m x) \cos (n y) d\left(\frac{1}{A-2 \cos (x)-2 \cos (y)}\right) d y \\
& =-m \frac{1}{4 \pi^{2}} \int_{0}^{2 \pi} \int_{0}^{2 \pi} \frac{\cos (m x) \cos (n y)}{A-2 \cos (x)-2 \cos (y)} d x d y=-m \tilde{g}_{m, n} .
\end{aligned}
$$

We obtain

$$
g_{m+1, n}=g_{m-1, n}+\frac{i m g_{m, n}}{t} .
$$

We also have

$$
i\left(s \tilde{g}_{0,0}-i\right)=-\left(\tilde{g}_{-1,0}+\tilde{g}_{1,0}+\tilde{g}_{0,-1}+\tilde{g}_{0,1}-4 \tilde{g}_{0,0}\right),
$$

and

$$
g_{m, n}=g_{n, m}, \quad g_{-m, n}=g_{m, n}, \quad g_{m,-n}=g_{m, n} .
$$

Now collecting (18), (26), (27) and (28), we can construct a system of ordinary differential equations to compute $g_{m, n}(t)$ in the domain $\{(m, n) \mid 0 \leq m \leq L, 0 \leq n \leq L\}$. The equations in the interior domain can be written as

$$
\begin{aligned}
& i \dot{g}_{m, n}=-\left(g_{m+1, n}+g_{m-1, n}+g_{m, n+1}+g_{m, n-1}-4 g_{m, n}\right), \\
& \left.g_{m, n}\right|_{t=0}=0, \quad \text { for } \quad\{(m, n) \mid 1 \leq m \leq L-1,1 \leq n \leq L-1\} .
\end{aligned}
$$


The lower boundary condition is

$$
\begin{aligned}
& i \dot{g}_{0, n}=-\left(g_{0, n+1}+g_{0, n-1}+2 g_{1, n}-4 g_{0, n}\right), \\
& \left.g_{0, n}\right|_{t=0}=0, \quad 1 \leq n \leq L-1 .
\end{aligned}
$$

Similarly, the left boundary condition reads

$$
\begin{aligned}
& i \dot{g}_{m, 0}=-\left(g_{m+1,0}+g_{m-1,0}+2 g_{m, 1}-4 g_{m, 0}\right), \\
& \left.g_{m, 0}\right|_{t=0}=0, \quad 1 \leq m \leq L-1 .
\end{aligned}
$$

By (26) and (28), one has the upper boundary condition

$$
\begin{aligned}
& i \dot{g}_{L, n}=-\left(\frac{i L g_{L, n}}{t}+g_{L, n+1}+g_{L, n-1}+2 g_{L-1, n}-4 g_{L, n}\right), \\
& \left.g_{L, n}\right|_{t=0}=0, \quad 1 \leq n \leq L-1 .
\end{aligned}
$$

In the same way, the right boundary condition writes

$$
\begin{aligned}
& i \dot{g}_{m, L}=-\left(\frac{i L g_{m, L}}{t}+g_{m+1, L}+g_{m-1, L}+2 g_{m, L-1}-4 g_{m, L}\right), \\
& \left.g_{m, L}\right|_{t=0}=0, \quad 1 \leq m \leq L-1 .
\end{aligned}
$$

By (18), we get the lower-left corner condition

$$
i \dot{g}_{0,0}=-\left(4 g_{0,1}-4 g_{0,0}\right),\left.\quad g_{0,0}\right|_{t=0}=i .
$$

Following a similar derivation, the other corner conditions can be obtained as

$$
\begin{array}{cc}
i \dot{g}_{L, 0}=-\left(\frac{i L g_{L, 0}}{t}+2 g_{L, 1}+2 g_{L-1,0}-4 g_{L, 0}\right), & \left.g_{L, 0}\right|_{t=0}=0, \\
i \dot{g}_{0, L}=-\left(\frac{i L g_{0, L}}{t}+2 g_{1, L}+2 g_{0, L-1}-4 g_{0, L}\right), & \left.g_{0, L}\right|_{t=0}=0, \\
i \dot{g}_{L, L}=-\left(\frac{2 i L g_{L, L}}{t}+2 g_{L, L-1}+2 g_{L-1, L}-4 g_{L, L}\right), & \left.g_{L, L}\right|_{t=0}=0 .
\end{array}
$$

In practice, to compute an accurate numerical approximation of $g_{m, n}(t)$, we use the Runge-Kutta 4 (RK4) scheme. If $u^{0}$ is known, we then compute $u^{1}$ by RK4 on a large enough domain, and use the zero boundary condition. If $u^{0}$ is compactly supported and the domain is large enough, the boundary points of $u^{1}$ are still 0 and we can use RK4 and closed relations to compute $u^{1}$ and so on. Let us also remark that other schemes than RK4 could be used, but of order larger than 2. Nevertheless, stability problems may arise and therefore the ODE solver must be carefully chosen. To illustrate the results, we compare the numerical and exact solutions for $g_{0,0}(t)$ in Fig. 2 . As the time step $\Delta t$ decreases, the approximation quality of $g_{m, n}(t)$ increases. The computed convergence rates of the numerical approximation of $g_{60,60}(12)$ and $g_{60,60}(36)$ are reported in Fig. 3. As expected, it is around 4. The algorithm is then able to efficiently and accurately compute $g_{m, n}(t)$ even for large values of the indices $m$ and $n$.

We also remark that the proposed approach extends to the $N$-dimensional Schrödinger equation, for which the single-source kernel function for the Schrödinger equation reads

$$
\tilde{g}_{m_{1}, m_{2}, \ldots m_{N}}(s)=\frac{1}{(2 \pi)^{N}} \int_{0}^{2 \pi} \int_{0}^{2 \pi} \cdots \int_{0}^{2 \pi} \frac{\exp \left(-i \sum_{\ell=1}^{N} m_{\ell} x_{\ell}\right)}{4-i s-2 \sum_{\ell=1}^{N} \cos \left(x_{\ell}\right)} d x_{1} \cdots d x_{N} .
$$

The recurrence relation is then

$$
g_{m_{1}, m_{2}, \ldots m_{N}}(t)=g_{m_{1}, m_{2}, \ldots m_{\ell}-1, \ldots m_{N}}(t)+\frac{i m_{\ell} g_{m_{1}, m_{2}, \ldots m_{N}}(t)}{t} .
$$

Therefore, one can also build an associated system of ordinary differential equations to calculate numerically $g_{m_{1}, m_{2}, \ldots m_{N}}(t)$. 


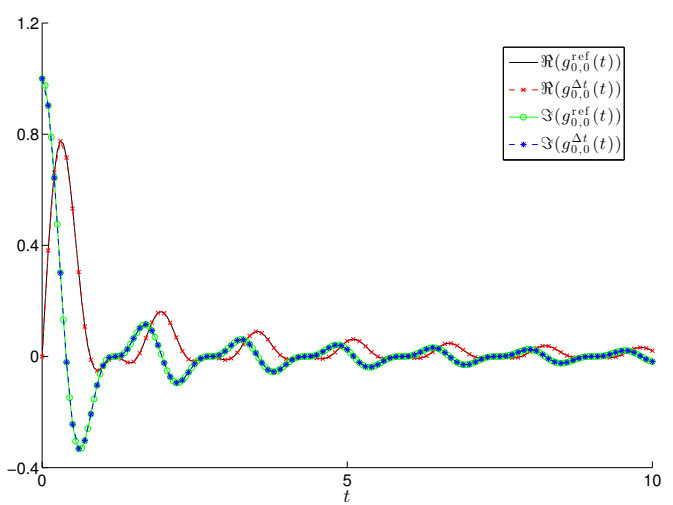

Figure 2: Numerical and reference solutions for $g_{0,0}(t)$ and the semi-discretized Schrödinger equation.
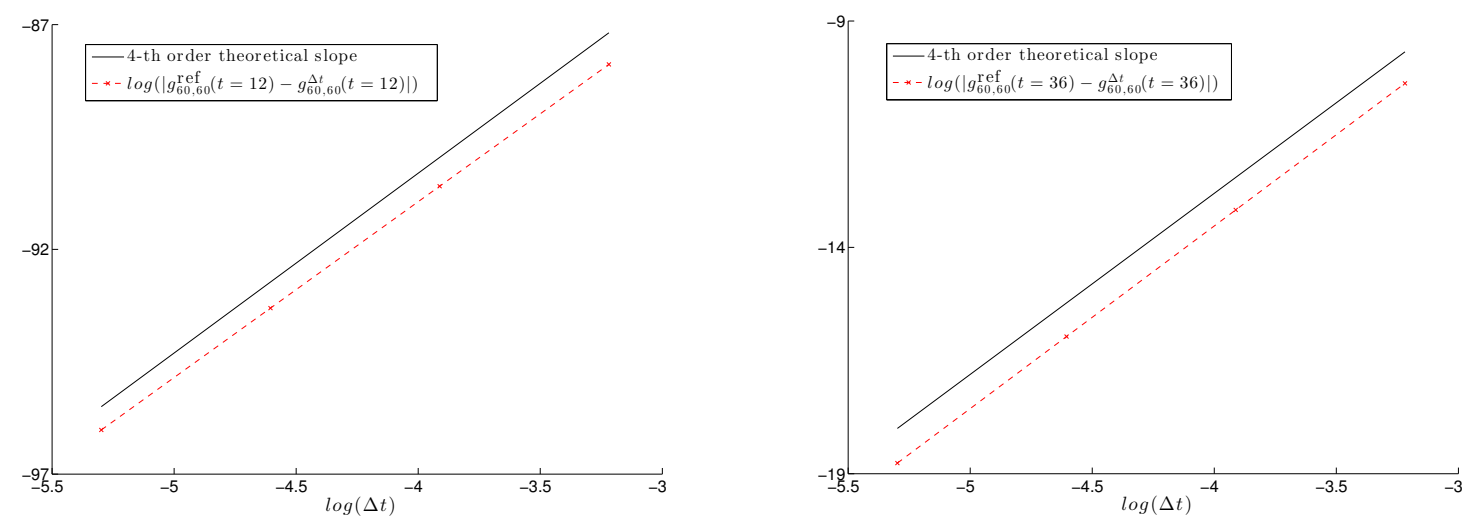

Figure 3: Theoretical and numerical slopes of the convergence rates for the approximation of $g_{60,60}(12)$ (left) and $g_{60,60}(36)$ (right).

\subsection{Extension to the heat equation}

We extend now the above method to the heat equation

$$
\begin{aligned}
& \left.\left.\frac{\partial}{\partial t} u=\left(\frac{\partial^{2}}{\partial x^{2}}+\frac{\partial^{2}}{\partial y^{2}}\right) u, \quad(\mathbf{x}, t) \text { in } \mathbb{R}^{2} \times\right] 0, T\right], \\
& u(\mathbf{x}, t=0)=0, \quad \mathbf{x} \in \Omega^{\mathrm{ext}}=\mathbb{R}^{2} \backslash \Omega^{\mathrm{int}} \\
& u(\mathbf{x}, \cdot) \rightarrow 0, \quad \text { for }\|\mathbf{x}\| \rightarrow+\infty
\end{aligned}
$$

We remark that the heat equation can be a transformed Black-Scholes equation. Hence, some applications in finance can also be considered from the present developments.

For $(L+1) \Delta x=a$, the semi-discretization of (45) gives

$$
\begin{aligned}
& \left.\left.\dot{u}_{m, n}=\left(u_{m+1, n}+u_{m-1, n}+u_{m, n+1}+u_{m, n-1}-4 u_{m, n}\right), \quad \text { in } \mathbb{Z}^{2} \times\right] 0, T\right], \\
& \left.u_{m, n}\right|_{t=0}=0, \quad \text { in } \quad(m, n) \in \mathbb{Z}^{2} \backslash\{(p, q) \mid 1 \leq p \leq L, 1 \leq q \leq L\}, \\
& u_{m, n} \rightarrow 0 \text { for }|m|,|n| \rightarrow+\infty
\end{aligned}
$$

If the computation of (48), (49) and (50) is confined within the finite domain $\{(m, n) \mid 0 \leq m \leq L+1,0 \leq n \leq L+1\}$, 
the boundary conditions read

$$
\begin{aligned}
& u_{I_{k}}(t)=\sum_{\ell=1}^{4 L-4} g_{I_{k}-I_{\ell}}(t) * \lambda_{I_{\ell}}(t), \quad 1 \leq k \leq 4 L-4, \\
& u_{J_{k}}(t)=\sum_{\ell=1}^{4 L-4} g_{J_{k}-I_{\ell}}(t) * \lambda_{I_{\ell}}(t), \quad 1 \leq k \leq 4 L,
\end{aligned}
$$

with

$$
g_{m, n}(t)=\frac{(m+n) !}{m ! n !} \frac{4 \sqrt{\pi}(t)^{m+n} e^{-4 t}}{2^{m+n+2} \Gamma\left(\frac{m+n+1}{2}\right) \Gamma\left(\frac{m+n}{2}+1\right)}{ }_{2} F_{3}\left(\begin{array}{c}
\frac{m+n+1}{2}, \frac{m+n}{2}+1 ; 4 t^{2} \\
m+1, n+1, m+n+1
\end{array}\right) .
$$

According to (25), one has

$$
g_{m+1, n}=g_{m-1, n}-\frac{m g_{m, n}}{t} .
$$

By the same procedure as in Section 2.1, a system of ODEs can be constructed to compute $g_{m, n}(t)$ in the domain $\{(m, n) \mid 0 \leq m \leq L, 0 \leq n \leq L\}$. The interior equations are

$$
\begin{aligned}
& \dot{g}_{m, n}=g_{m+1, n}+g_{m-1, n}+g_{m, n+1}+g_{m, n-1}-4 g_{m, n}, \quad \text { in } \quad\{(m, n) \mid 1 \leq m \leq L-1,1 \leq n \leq L-1\}, \\
& \left.g_{m, n}\right|_{t=0}=0, \quad \text { in } \quad\{(m, n) \mid 1 \leq m \leq L-1,1 \leq n \leq L-1\} .
\end{aligned}
$$

Finally, the corner and boundary conditions can be obtained by extension. The values of $g_{m, n}(t)$ are computed accurately by RK4. For $t \in[0,10]$, we report in Fig. 4 (left) the approximate solution of $g_{0,0}(t)$ and the corresponding exact solutions which are close. In Fig. 4 (right), we display the convergence rate of the approximation of $g_{60,60}(60)$.
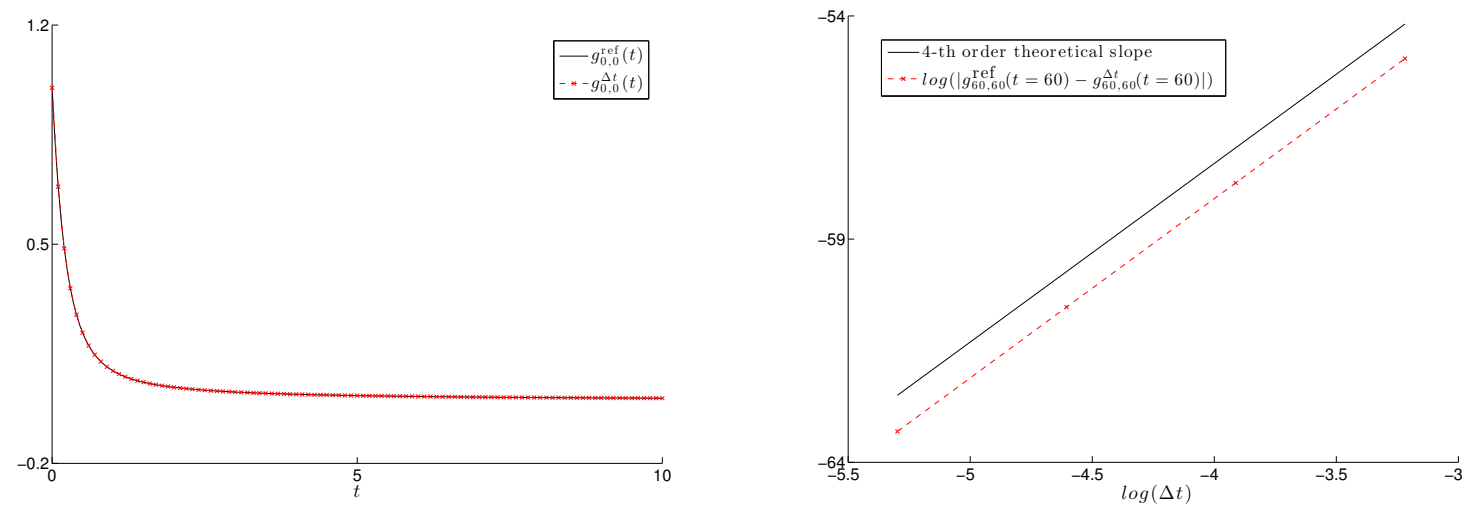

Figure 4: Left: numerical and reference solutions of $g_{0,0}(t)$ for the semi-discretized heat equation. Right: Theoretical and numerical slopes of the convergence rates for the approximation of $g_{60,60}(60)$.

\section{Numerical discretization}

In this section, we design the corresponding discretization of the reduced ordinary differential problem with the accurate artificial boundary condition. For conciseness, we only give the discretized finite difference scheme for the Schrödinger equation. The discretization for the reduced heat equation can easily be transposed. Following subsection 2.1, we adopt the Crank-Nicolson scheme [30] for the time integration in (4)

$$
\begin{gathered}
u_{m, n}^{k+1}-\frac{i \Delta t}{2}\left(u_{m+1, n}^{k+1}+u_{m-1, n}^{k+1}+u_{m, n+1}^{k+1}+u_{m, n-1}^{k+1}-4 u_{m, n}^{k+1}\right)= \\
u_{m, n}^{k}+\frac{i \Delta t}{2}\left(u_{m+1, n}^{k}+u_{m-1, n}^{k}+u_{m, n+1}^{k}+u_{m, n-1}^{k}-4 u_{m, n}^{k}\right) .
\end{gathered}
$$


After the computation of $g_{m, n}(t)$, the convolutions in (22) are performed through the trapezoidal rule as follows

$$
u_{I_{p}}^{k+1}=\sum_{\ell=1}^{4 L-4}\left(\frac{\Delta t}{2} g_{I_{p}-I_{\ell}}^{0} \lambda_{I_{\ell}}^{k+1}+\Delta t \sum_{\alpha=1}^{k} g_{I_{p}-I_{\ell}}^{k+1-\alpha} \lambda_{I_{\ell}}^{\alpha}+\frac{\Delta t}{2} g_{I_{p}-I_{\ell}}^{k+1} \lambda_{I_{\ell}}^{0}\right),
$$

for $p=1, \ldots, 4 L-4$ and where $g_{m, n}^{\alpha}$ denotes the fourth-order Runge-Kutta approximation of $g_{m, n}(\alpha \Delta t)$ for the time step $\Delta t$. Rewriting the above equation into the matrix form leads to

$$
\left(\begin{array}{c}
u_{I_{1}}^{k+1} \\
u_{I_{2}}^{k+1} \\
\vdots \\
u_{I_{4 L-4}}^{k+1}
\end{array}\right)=\Delta t\left(\begin{array}{cccc}
\frac{g_{I_{1}-I_{1}}^{0}}{2} & \frac{g_{I_{1}-I_{2}}^{0}}{2} & \ldots & \frac{g_{I_{1}-I_{L L}-4}^{0}}{2} \\
\frac{g_{I_{2}-I_{1}}}{2} & \frac{g_{I_{2}-I_{2}}}{2} & \ldots & \frac{g_{I_{2}-I_{L L}-4}}{2} \\
\vdots & \vdots & \vdots & \vdots \\
\frac{g_{I_{L L}-I_{1}}^{0}}{2} & \frac{g_{I_{4 L-4}-I_{2}}^{0}}{2} & \ldots & \frac{g_{I_{4 L-4}-I_{4 L-4}}^{0}}{2}
\end{array}\right)\left(\begin{array}{c}
\lambda_{I_{1}}^{k+1} \\
\lambda_{I_{2}}^{k+1} \\
\vdots \\
\lambda_{I_{4 L-4}}^{k+1}
\end{array}\right)+\left(\begin{array}{c}
\Delta t \sum_{\ell=1}^{4 L-4} g_{I_{1}-I_{\ell}}^{k+1} \lambda_{I_{\ell}}^{0}+\Delta t \sum_{\ell=1}^{4 L-4} \sum_{\alpha=1}^{k} g_{I_{1}-I_{\ell}}^{k+1-\alpha} \lambda_{I_{\ell}}^{\alpha} \\
\frac{\Delta t}{2} \sum_{\ell=1}^{4 L-4} g_{I_{2}-I_{\ell}}^{k+1} \lambda_{I_{\ell}}^{0}+\Delta t \sum_{\ell=1}^{4 L-4} \sum_{\alpha=1}^{k} g_{I_{2}-I_{\ell}}^{k+1-\alpha} \lambda_{I_{\ell}}^{\alpha} \\
\vdots \\
\frac{\Delta t}{2} \sum_{\ell=1}^{4 L-4} g_{I_{4 L-4}-I_{\ell}}^{k+1} \lambda_{I_{\ell}}^{0}+\Delta t \sum_{\ell=1}^{4 L-4} \sum_{\alpha=1}^{k} g_{I_{4 L-4}-I_{\ell}}^{k+1-\alpha} \lambda_{I_{\ell}}^{\alpha}
\end{array}\right) .
$$

Similarly one gets

$$
\left(\begin{array}{c}
u_{J_{1}}^{k+1} \\
u_{J_{2}}^{k+1} \\
\vdots \\
u_{J_{4 L}}^{k+1}
\end{array}\right)=\Delta t\left(\begin{array}{cccc}
\frac{g_{J_{1}-I_{1}}^{0}}{2} & \frac{g_{J_{1}-I_{2}}^{0}}{2} & \ldots & \frac{g_{J_{1}-I_{4 L}-4}^{0}}{2} \\
\frac{g_{J_{2}-I_{1}}^{0}}{2} & \frac{g_{J_{2}-I_{2}}}{2} & \ldots & \frac{g_{J_{2}-I_{4 L}-4}}{2} \\
\vdots & \vdots & \vdots & \vdots \\
\frac{g_{J_{L L}-I_{1}}^{0}}{2} & \frac{g_{J_{4 L}-I_{2}}^{0}}{2} & \ldots & \frac{g_{J_{4 L}-I_{4 L}-4}}{2}
\end{array}\right)\left(\begin{array}{c}
\lambda_{I_{1}}^{k+1} \\
\lambda_{I_{2}}^{k+1} \\
\vdots \\
\lambda_{I_{4 L-4}}^{k+1}
\end{array}\right)+\left(\begin{array}{c}
\frac{\Delta t}{2} \sum_{\ell=1}^{4 L-4} g_{J_{1}-I_{\ell}}^{k+1} \lambda_{I_{\ell}}^{0}+\Delta t \sum_{\ell=1}^{4 L-4} \sum_{\alpha=1}^{k} g_{J_{1}-I_{\ell}}^{k+1-\alpha} \lambda_{I_{\ell}}^{\alpha} \\
\frac{\Delta t}{2} \sum_{\ell=1}^{4 L-4} g_{J_{2}-I_{\ell}}^{k+1} \lambda_{L_{\ell}}^{0}+\Delta t \sum_{\ell=1}^{4 L-4} \sum_{\alpha=1}^{k} g_{J_{2}-I_{\ell}}^{k+1-\alpha} \lambda_{I_{\ell}}^{\alpha} \\
\vdots \\
\frac{\Delta t}{2} \sum_{\ell=1}^{4 L-4} g_{J_{4 L}-I_{\ell}}^{k+1} \lambda_{I_{\ell}}^{0}+\Delta t \sum_{\ell=1}^{4 L-4} \sum_{\alpha=1}^{k} g_{J_{4 L}-I_{\ell}}^{k+1-\alpha} \lambda_{I_{\ell}}^{\alpha}
\end{array}\right) .
$$

In addition, since $g_{m, n}(0)=0$ for $|m|+|n|>0$, one deduces

$$
\left(\begin{array}{c}
u_{J_{1}}^{k+1} \\
u_{J_{2}}^{k+1} \\
\vdots \\
u_{J_{4 L}}^{k+1}
\end{array}\right)=\left(\begin{array}{c}
\frac{\Delta t}{2} \sum_{\ell=1}^{4 L-4} g_{J_{1}-I_{\ell}}^{k+1} \lambda_{I_{\ell}}^{0}+\Delta t \sum_{\ell=1}^{4 L-4} \sum_{\alpha=1}^{k} g_{J_{I^{\prime}}-I_{\ell}}^{k+1-\alpha} \lambda_{I_{\ell}}^{\alpha} \\
\frac{\Delta t}{2} \sum_{\ell=1}^{4 L-4} g_{J_{2}-I_{\ell}}^{k+1} \lambda_{L_{\ell}}^{0}+\Delta t \sum_{\ell=1}^{4 L-4} \sum_{\alpha=1}^{k} g_{J_{2}-I_{\ell}}^{k+1} \lambda_{I_{\ell}}^{\alpha} \\
\vdots \\
\frac{\Delta t}{2} \sum_{\ell=1}^{4 L-4} g_{J_{4 L}-I_{\ell}}^{k+1} \lambda_{I_{\ell}}^{0}+\Delta t \sum_{\ell=1}^{4 L-4} \sum_{\alpha=1}^{k} g_{J_{4 L}-I_{\ell}}^{k+1-\alpha} \lambda_{I_{\ell}}^{\alpha}
\end{array}\right) .
$$

We denote the vector $\left[u_{I_{1}}^{k}, u_{I_{2}}^{k} . . u_{I_{4 L-4}}^{k}\right]^{T}$ by $u_{I}^{k},\left[\lambda_{I_{1}}^{k}, \lambda_{I_{2}}^{k} \ldots \lambda_{I_{4 L-4}}^{k}\right]^{T}$ by $\lambda_{I}^{k}$ and $\left[u_{J_{1}}^{k}, u_{J_{2}}^{k} . . u_{J_{4 L}}^{k}\right]^{T}$ by $u_{J}^{k}$. Therefore, we can recast systems (58) and (60) through a matrix $\mathbf{A}_{1}$, and two vectors $R_{1}^{k+1}$, and $R_{2}^{k+1}$ as

$$
\begin{aligned}
& u_{I}^{k+1}=\mathbf{A}_{1} \lambda_{I}^{k+1}+R_{1}^{k+1}, \\
& u_{J}^{k+1}=R_{2}^{k+1} .
\end{aligned}
$$

Now one can use (61) with (56). All the points $(m, n)$, for $1 \leq m, n \leq L$, are indexed following

$$
N_{k}=\left(\frac{k-\bmod (k, L)}{L}+1, \bmod (k, L)\right)
$$

for $1 \leq k \leq L^{2}$. If $I_{k}=(m, n)$, we define $I^{-1}(m, n)=k$ (and similarly for $J_{k}=(m, n)$ and $N_{k}=(m, n)$ ). The vector $\left[u_{N_{1}}^{k}, u_{N_{2}}^{k} . . u_{N_{L^{2}}}^{k}\right]^{T}$ is denoted by $u_{N^{*}}^{k}$. If we have all the data until the $k$-th time step, $\lambda_{I_{\ell}}^{\alpha}$ for $(1 \leq \ell \leq 4 L-4$ and $0 \leq \alpha \leq k)$ and $u_{m, n}^{k}$ for $0 \leq m, n \leq L$, then we can compute $u_{J}^{k+1}=R_{2}^{k+1}$. We can also rewrite (56) as $\mathbf{B} u_{N}^{k+1}=F^{k+1}$, where $\mathbf{B}$ is a $L^{2} \times L^{2}$ matrix defined for computing the values $u_{m, n}^{k+1}$ for $1 \leq m, n \leq L$ and $F^{k+1}$ is the right-hand side. More precisely, for $(m, n)$, with $2 \leq m, n \leq L-1$, and from (56) we have

$$
\begin{array}{lr}
\mathbf{B}_{N^{-1}(m, n), N^{-1}(m, n)}=1+2 i \Delta t, & \mathbf{B}_{N^{-1}(m, n), N^{-1}(m-1, n)}=-\frac{i \Delta t}{2}, \\
\mathbf{B}_{N^{-1}(m, n), N^{-1}(m+1, n)}=-\frac{i \Delta t}{2}, & \mathbf{B}_{N^{-1}(m, n), N^{-1}(m, n+1)}=-\frac{i \Delta t}{2}, \\
\mathbf{B}_{N^{-1}(m, n), N^{-1}(m, n-1)}=-\frac{i \Delta t}{2}, & \\
F_{N^{-1}(m, n)}^{k+1}=u_{m, n}^{k}+\frac{i \Delta t}{2}\left(u_{m+1, n}^{k}+u_{m-1, n}^{k}+u_{m, n+1}^{k}+u_{m, n-1}^{k}-4 u_{m, n}^{k}\right) . &
\end{array}
$$


For $(m, n)=(1,1)$, we also can write

$$
(1+2 i \Delta t) u_{1,1}^{k+1}-\frac{i \Delta t}{2}\left(u_{2,1}^{k+1}+u_{1,2}^{k+1}\right)=u_{1,1}^{k}+\frac{i \Delta t}{2} u_{1,0}^{k+1}+\frac{i \Delta t}{2} u_{0,1}^{k+1}+\frac{i \Delta t}{2}\left(u_{2,1}^{k}+u_{0,1}^{k}+u_{1,2}^{k}+u_{1,0}^{k}-4 u_{1,1}^{k}\right),
$$

and by (61) we have

$$
\begin{gathered}
\mathbf{B}_{N^{-1}(1,1), N^{-1}(1,1)}=1+2 i \Delta t, \quad \mathbf{B}_{N^{-1}(1,1), N^{-1}(1,2)}=-\frac{i \Delta t}{2}, \quad \mathbf{B}_{N^{-1}(1,1), N^{-1}(2,1)}=-\frac{i \Delta t}{2}, \\
F_{N^{-1}(1,1)}^{k+1}=u_{1,1}^{k}+\frac{i \Delta t}{2} u_{1,0}^{k+1}+\frac{i \Delta t}{2} u_{0,1}^{k+1}+\frac{i \Delta t}{2}\left(u_{2,1}^{k}+u_{0,1}^{k}+u_{1,2}^{k}+u_{1,0}^{k}-4 u_{1,1}^{k}\right) \\
=\frac{i \Delta t}{2}\left(R_{2}^{k+1}\right)_{J^{-1}(1,0)}+\frac{i \Delta t}{2}\left(R_{2}^{k+1}\right)_{J^{-1}(0,1)}+u_{1,1}^{k}+\frac{i \Delta t}{2}\left(u_{2,1}^{k}+u_{0,1}^{k}+u_{1,2}^{k}+u_{1,0}^{k}-4 u_{1,1}^{k}\right) .
\end{gathered}
$$

We can deal with the points $(m, n)=(1, L),(L, 1)$ and $(L, L)$ similarly to the point $(1,1)$. For $(m, n)=(1, \ell)$, for $2 \leq \ell \leq L-1$, one gets

$$
(1+2 i \Delta t) u_{1, \ell}^{k+1}-\frac{i \Delta t}{2}\left(u_{1, \ell-1}^{k+1}+u_{1, \ell+1}^{k+1}+u_{2, \ell}^{k+1}\right)=u_{1, \ell}^{k}+\frac{i \Delta t}{2} u_{0, \ell}^{k+1}+\frac{i \Delta t}{2}\left(u_{1, \ell+1}^{k}+u_{1, \ell-1}^{k}+u_{2, \ell}^{k}+u_{0, \ell}^{k}-4 u_{1, \ell}^{k}\right),
$$

which means

$$
\begin{array}{crl}
\mathbf{B}_{N^{-1}(1, \ell), N^{-1}(1, \ell)}=1+2 i \Delta t, & \mathbf{B}_{N^{-1}(1, \ell), N^{-1}(1, \ell-1)}=-\frac{i \Delta t}{2}, \\
\mathbf{B}_{N^{-1}(1, \ell), N^{-1}(1, \ell+1)}=-\frac{i \Delta t}{2}, & \mathbf{B}_{N^{-1}(1, \ell), N^{-1}(2, \ell)}=-\frac{i \Delta t}{2}, \\
F_{N^{-1}(1, \ell)}^{k+1}=\frac{i \Delta t}{2}\left(R_{2}^{k+1}\right)_{J^{-1}(0, \ell)}+u_{1, \ell}^{k}+\frac{i \Delta t}{2}\left(u_{1, \ell+1}^{k}+u_{1, \ell-1}^{k}+u_{2, \ell}^{k}+u_{0, \ell}^{k}-4 u_{1, \ell}^{k}\right) .
\end{array}
$$

The points $(m, n)=(\ell, 1),(L, \ell)$ and $(\ell, L)$, for $2 \leq \ell \leq L-1$, can be treated as $(1, \ell)$. The other coefficients of $\mathbf{B}$ are equal to 0 . Now we get $u_{N}^{k+1}$ and all $u_{m, n}^{k+1}$ can be computed for $1 \leq m, n \leq L$. To update $\lambda_{I}^{k+1}$, one uses the relation

$$
\lambda_{I}^{k+1}=\left(\mathbf{A}_{1}\right)^{-1}\left(u_{I}^{k+1}-R_{1}^{k+1}\right)
$$

and the $\lambda_{I}^{k+1}$ are next used to build $R_{2}^{k+2}$.

\section{Numerical results}

\subsection{The semi-discretized Schrödinger equation}

Let us consider the semi-discretized Schrödinger equation (4) with the discrete initial data

$$
u_{m, n}(0)=\exp \left(-\left(\frac{m-23.5}{6}\right)^{2}-\left(\frac{n-23.5}{6}\right)^{2}-i \frac{5(m-23.5)}{6}+i \frac{5(n-23.5)}{6}\right) .
$$

This wave strikes the boundary allowing therefore to analyze the quality of the boundary condition to avoid some spurious reflection that would come back into the computational domain. We fix the spatial grid to $L=46$. The numerical reference solution $u^{\text {ref }}$ is calculated for a very small time step and on a large domain so that there is no interacting wave with the boundary. We report at time $t$ the absolute $\ell^{2}$ - and $\ell^{\infty}$-errors between the numerical solution $u^{\Delta t}(t):=\left(u_{m, n}^{\Delta t}\right)_{1 \leq m, n \leq L}$ for a time step $\Delta t$ and the reference solution $u^{\mathrm{ref}}(t)$, and defined by

$$
E_{\Delta t}^{2}(t):=\sqrt{\sum_{m=1}^{L} \sum_{n=1}^{L}\left|u_{m, n}^{\mathrm{ref}}(t)-u_{m, n}^{\Delta t}(t)\right|^{2}}, \quad E_{\Delta t}^{\infty}(t):=\max _{1 \leq m, n \leq L}\left|u_{m, n}^{\mathrm{ref}}(t)-u_{m, n}^{\Delta t}(t)\right| .
$$

The evolution of the numerical solution with the proposed boundary condition is reported in Fig. 5 for the times $t=0$, 12,14 and 36. We do not observe any reflection at the corners. The errors $\left|u_{m, n}^{\text {ref }}(14)-u_{m, n}^{\Delta t}(14)\right|\left(\right.$ with $\left.\Delta t=2 \times 10^{-2}\right)$ on the grid are displayed in Fig. 6 (left) and with a maximum of the order of $7 \times 10^{-5}$. For completeness, we report $u_{1,46}^{\text {ref }}(t)-u_{1,46}^{\Delta t}(t)$ (with $\Delta t=2 \times 10^{-2}$ ) on Fig. 6 (right). In Tables 1 and 2, we provide respectively the errors $E_{\Delta t}^{2}(t)$ and $E_{\Delta t}^{\infty}(t)$, for $t=2,12,14,36$, and for various time steps $\Delta t$. We clearly observe that the scheme is at least second-order in time which is coherent with the expected second-order accuracy of the Crank-Nicolson scheme (the numerical rate of convergence are obtained as the least-square approximations of the pointwise rates). 

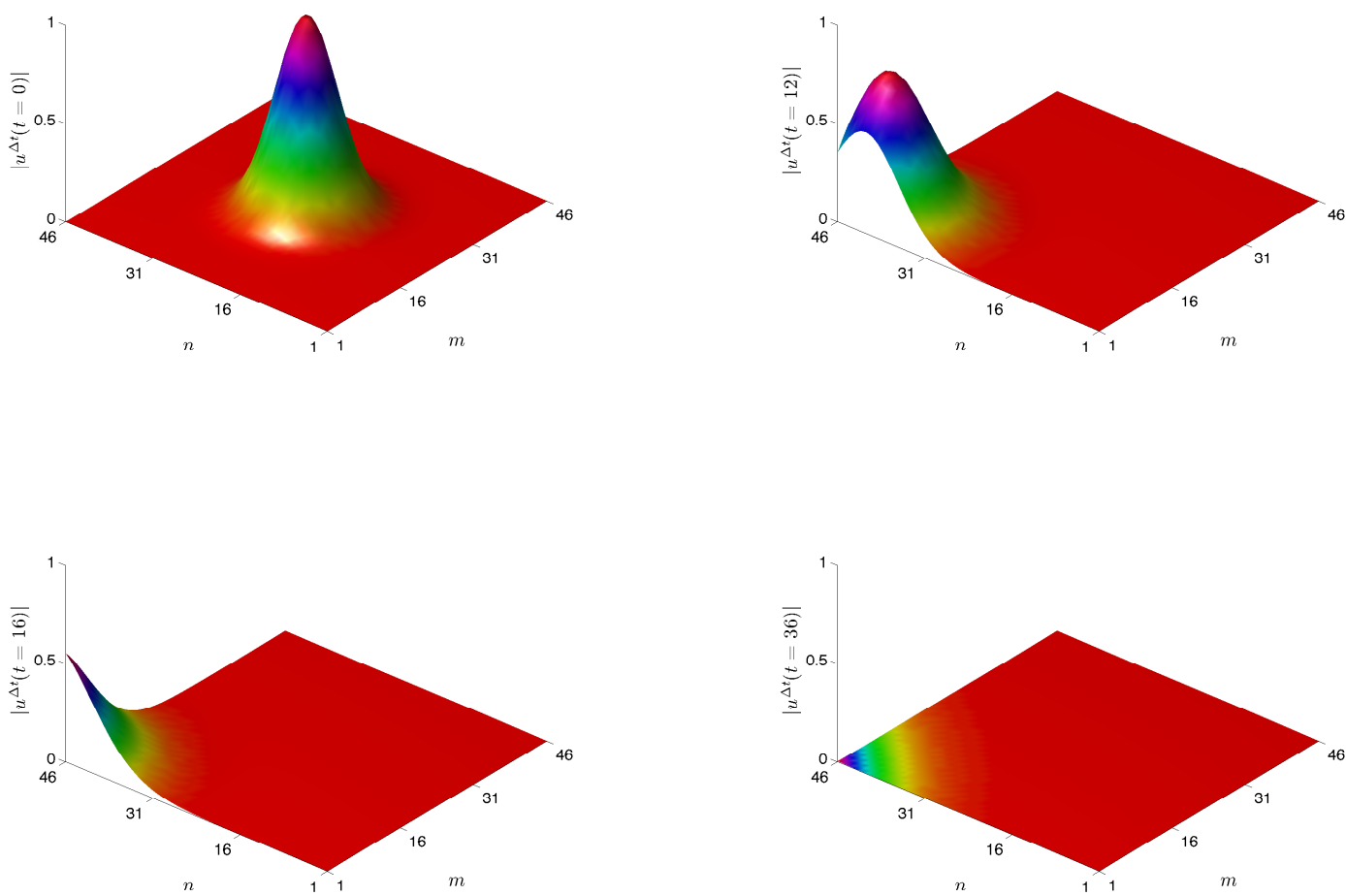

Figure 5: Amplitude of the numerical solution $\left|u^{\Delta t}\right|$ (with $\Delta t=2 \times 10^{-2}$ ) of the Schrödinger equation on the $46 \times 46$ square computational domain at different times $t$ (upper-left for $t=0$; upper-right for $t=12$; lower-left for $t=14$; lower-right for $t=36$ ).
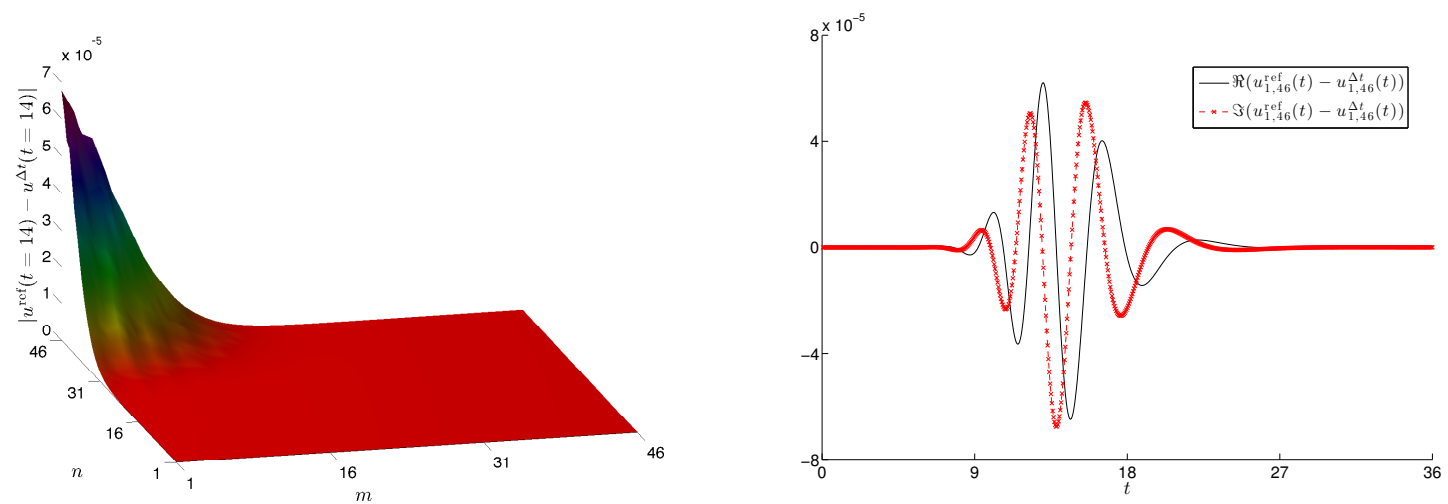

Figure 6: Left: absolute error $\left|u_{m, n}^{\text {ref }}(14)-u_{m, n}^{\Delta t}(14)\right|\left(\right.$ with $\left.\Delta t=2 \times 10^{-2}\right)$ for the Schrödinger equation on the $46 \times 46$ square computational domain. Right: error on the real and imaginary parts at the upper left corner.

\subsection{The semi-discretized heat equation}

We consider now the case of the semi-discretized heat equation (48) with the initial data uniformly set equal to 10 on a $8 \times 8$ sub square lattice, and zero otherwise. We choose a $10 \times 10$ computational domain, which is small enough to test the accuracy of the boundary condition. We report on Fig. 7 and for $\Delta t=2 \times 10^{-2}$ the numerical solution in the truncated domain. We can see that there is no corner reflection. The errors $\left|u_{m, n}^{\mathrm{ref}}(40)-u_{m, n}^{\Delta t}(40)\right|$ on the grid 


\begin{tabular}{|c|c|c|c|c|}
\hline$E_{\Delta t}^{2}(t)$ & $t=2$ & $t=12$ & $t=14$ & $t=36$ \\
\hline$\Delta t=8 \times 10^{-2}$ & $2.98 \times 10^{-2}$ & $1.31 \times 10^{-1}$ & $8.37 \times 10^{-2}$ & $9.00 \times 10^{-3}$ \\
\hline$\Delta t=5 \times 10^{-2}$ & $1.16 \times 10^{-2}$ & $5.11 \times 10^{-2}$ & $3.25 \times 10^{-2}$ & $3.58 \times 10^{-3}$ \\
\hline$\Delta t=4 \times 10^{-2}$ & $7.44 \times 10^{-3}$ & $3.26 \times 10^{-2}$ & $2.07 \times 10^{-2}$ & $2.30 \times 10^{-3}$ \\
\hline$\Delta t=2 \times 10^{-2}$ & $1.84 \times 10^{-3}$ & $8.05 \times 10^{-3}$ & $5.11 \times 10^{-3}$ & $5.79 \times 10^{-4}$ \\
\hline$\Delta t=1 \times 10^{-2}$ & $4.38 \times 10^{-4}$ & $1.92 \times 10^{-3}$ & $1.22 \times 10^{-3}$ & $1.45 \times 10^{-4}$ \\
\hline$\Delta t=5 \times 10^{-3}$ & $8.76 \times 10^{-5}$ & $3.84 \times 10^{-4}$ & $2.44 \times 10^{-4}$ & $3.63 \times 10^{-5}$ \\
\hline rate & 2.09 & 2.09 & 2.09 & 2.00 \\
\hline
\end{tabular}

Table 1: Error $E_{\Delta t}^{2}(t)$ at different times for various time steps $\Delta t$ (Schrödinger equation).

\begin{tabular}{|c|c|c|c|c|}
\hline$E_{\Delta t}^{\infty}(t)$ & $t=2$ & $t=12$ & $t=14$ & $t=36$ \\
\hline$\Delta t=8 \times 10^{-2}$ & $3.86 \times 10^{-3}$ & $1.94 \times 10^{-2}$ & $2.08 \times 10^{-2}$ & $3.47 \times 10^{-4}$ \\
\hline$\Delta t=5 \times 10^{-2}$ & $1.51 \times 10^{-3}$ & $7.56 \times 10^{-3}$ & $8.10 \times 10^{-3}$ & $1.38 \times 10^{-4}$ \\
\hline$\Delta t=4 \times 10^{-2}$ & $9.66 \times 10^{-4}$ & $4.83 \times 10^{-3}$ & $5.17 \times 10^{-3}$ & $8.88 \times 10^{-5}$ \\
\hline$\Delta t=2 \times 10^{-2}$ & $2.39 \times 10^{-4}$ & $1.19 \times 10^{-3}$ & $1.28 \times 10^{-3}$ & $2.24 \times 10^{-5}$ \\
\hline$\Delta t=1 \times 10^{-2}$ & $5.69 \times 10^{-5}$ & $2.84 \times 10^{-4}$ & $3.05 \times 10^{-4}$ & $5.61 \times 10^{-6}$ \\
\hline$\Delta t=5 \times 10^{-3}$ & $1.14 \times 10^{-5}$ & $5.71 \times 10^{-5}$ & $6.22 \times 10^{-5}$ & $1.40 \times 10^{-6}$ \\
\hline rate & 2.09 & 2.09 & 2.08 & 1.99 \\
\hline
\end{tabular}

Table 2: $E_{\Delta t}^{\infty}(t)$ at different times for various time steps $\Delta t$ (Schrödinger equation).

are displayed in Fig 8. The reference solution is calculated over a much larger domain and again with a very small time step. The errors in $\ell_{2}$ - and $\ell_{\infty}$-norms with respect to $\Delta t$ are reported in Tables 3 and 4 , respectively. Again, a second-order convergence is observed.

\begin{tabular}{|c|c|c|c|c|}
\hline$E_{\Delta t}^{2}(t)$ & $t=2$ & $t=4$ & $t=10$ & $t=40$ \\
\hline$\Delta t=8 \times 10^{-2}$ & $2.61 \times 10^{-2}$ & $3.15 \times 10^{-2}$ & $3.38 \times 10^{-2}$ & $1.61 \times 10^{-2}$ \\
\hline$\Delta t=5 \times 10^{-2}$ & $1.01 \times 10^{-2}$ & $1.23 \times 10^{-2}$ & $1.31 \times 10^{-2}$ & $6.25 \times 10^{-3}$ \\
\hline$\Delta t=4 \times 10^{-2}$ & $6.49 \times 10^{-3}$ & $7.84 \times 10^{-3}$ & $8.40 \times 10^{-3}$ & $3.99 \times 10^{-3}$ \\
\hline$\Delta t=2 \times 10^{-2}$ & $1.62 \times 10^{-3}$ & $1.96 \times 10^{-3}$ & $2.10 \times 10^{-3}$ & $7.97 \times 10^{-4}$ \\
\hline$\Delta t=1 \times 10^{-2}$ & $4.05 \times 10^{-4}$ & $4.89 \times 10^{-4}$ & $5.24 \times 10^{-4}$ & $2.49 \times 10^{-4}$ \\
\hline$\Delta t=5 \times 10^{-3}$ & $1.01 \times 10^{-4}$ & $1.22 \times 10^{-4}$ & $1.31 \times 10^{-4}$ & $6.23 \times 10^{-5}$ \\
\hline rate & 2.00 & 2.00 & 2.00 & 2.00 \\
\hline
\end{tabular}

Table 3: $E_{\Delta t}^{2}(t)$ at different times for various time steps $\Delta t$ (heat equation).

\begin{tabular}{|c|c|c|c|c|}
\hline$E_{\Delta t}^{\infty}(t)$ & $t=2$ & $t=4$ & $t=10$ & $t=40$ \\
\hline$\Delta t=8 \times 10^{-2}$ & $3.99 \times 10^{-3}$ & $4.53 \times 10^{-3}$ & $3.68 \times 10^{-3}$ & $1.75 \times 10^{-3}$ \\
\hline$\Delta t=5 \times 10^{-2}$ & $1.55 \times 10^{-3}$ & $1.76 \times 10^{-3}$ & $1.43 \times 10^{-3}$ & $6.81 \times 10^{-4}$ \\
\hline$\Delta t=4 \times 10^{-2}$ & $9.93 \times 10^{-4}$ & $1.13 \times 10^{-3}$ & $9.15 \times 10^{-4}$ & $4.35 \times 10^{-4}$ \\
\hline$\Delta t=2 \times 10^{-2}$ & $2.48 \times 10^{-4}$ & $2.81 \times 10^{-4}$ & $2.28 \times 10^{-4}$ & $1.09 \times 10^{-4}$ \\
\hline$\Delta t=1 \times 10^{-2}$ & $6.24 \times 10^{-5}$ & $7.03 \times 10^{-5}$ & $5.71 \times 10^{-5}$ & $2.72 \times 10^{-5}$ \\
\hline$\Delta t=5 \times 10^{-3}$ & $1.59 \times 10^{-5}$ & $1.76 \times 10^{-5}$ & $1.43 \times 10^{-5}$ & $6.79 \times 10^{-6}$ \\
\hline rate & 1.99 & 2.00 & 2.00 & 2.00 \\
\hline
\end{tabular}

Table 4: $E_{\Delta t}^{\infty}(t)$ at different times for various time steps $\Delta t$ (heat equation). 

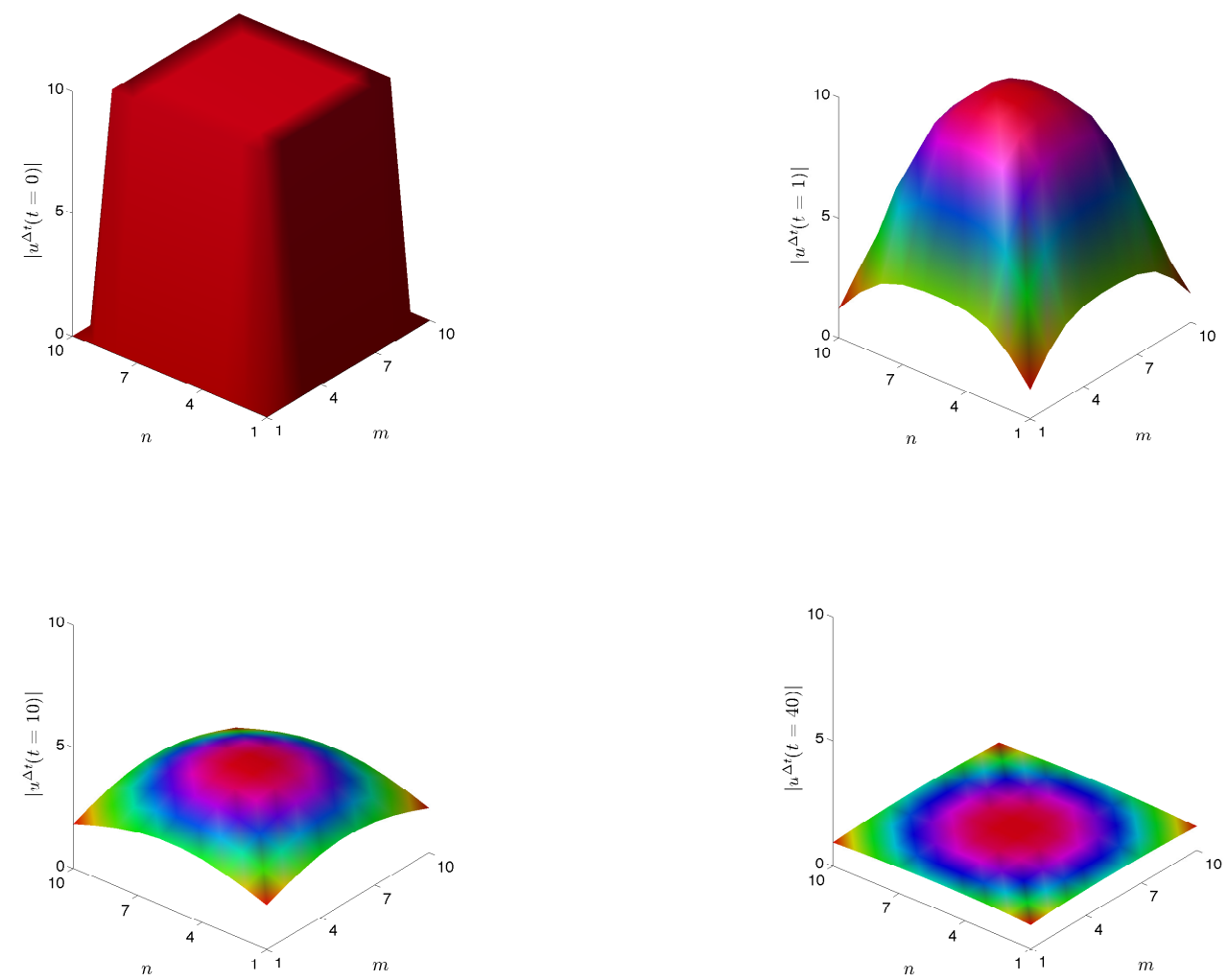

Figure 7: Amplitude of the numerical solution $\left|u^{\Delta t}\right|$ (with $\Delta t=2 \times 10^{-2}$ ) of the heat equation on the $10 \times 10$ square computational domain at different times $t$ (upper-left for $t=0$; upper-right for $t=1$; lower-left for $t=10$; lower-right for $t=40$ ).

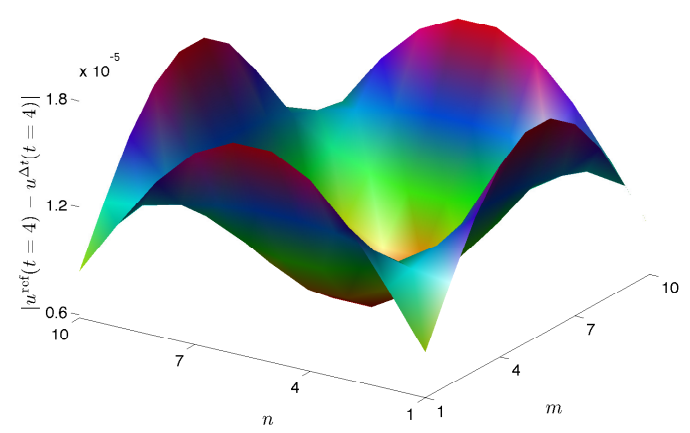

Figure 8: Absolute error $\left|u_{m, n}^{\text {ref }}(4)-u_{m, n}^{\Delta t}(4)\right|\left(\right.$ with $\Delta t=2 \times 10^{-2}$ ) for the heat equation on the $10 \times 10$ square computational domain.

\section{Conclusion}

In this paper, we investigated the accurate numerical solution of the semi-discretized linear Schrödinger equation by introducing an artificial boundary condition on a rectangular domain to bound the computational domain. The accurate evaluation of the boundary condition is realized by means of some recurrence relations involving the discrete Green's functions. As a result, the corner reflection is fully eliminated. We provide some numerical results that 
illustrate the second-order accuracy of the proposed scheme with some details about the implementation. Closely related artificial boundary conditions are applied to the heat equation and some numerical simulations show that they are still reflection-free.

Concerning the perspectives, some questions regarding the theoretical and numerical aspects of these boundary conditions remain to be answered. For example, the rigorous proof of the numerical stability and estimates of the convergence rate must still be obtained. Finally, fast algorithms for the boundary conditions also need to be explored in details to accelerate the evaluation of the transparent operator. These questions will be addressed in a forthcoming paper.

\section{Acknowledgements}

This study is partially supported by the National Natural Science Foundation of China under contract numbers 11272009, 11521202 and 11502028. X. Antoine thanks the support of the LIASFMA (funding from the University of Lorraine) and the French ANR grant Bond (ANR-13-BS01-0009-01).

\section{References.}

[1] E. Schrödinger, An undulatory theory of the mechanics of atoms and molecules, Physical Review. 28(6) (1926) 1049.

[2] D. Craig and T. Thirunamachandran, Molecular Quantum Electrodynamics, Academic Press, 321 (1984) 557.

[3] R. Hazeltine and F. Waelbroeck, The Framework of Plasma Physics, Perseus Books, (2004).

[4] Ö. Yilmaz, S. Doherty, Seismic Data Analysis: Processing, Inversion, and Interpretation of Seismic Data, Society of Exploration Geophysicists, (2001).

[5] J. Senior, Optical Fiber Communications: Principles and Practice, Prentice Hall, Extended and Updated 2nd Ed., (1992).

[6] J. Ferziger and M Peric, Computational Methods for Fluid Dynamics, Physics Today, 50(3) (2012) 80.

[7] F. Black and M. Scholes, The valuation of options and corporate liability, Journal of Political Economy, 81(3) (1973) 637.

[8] B. Engquist and A. Majda, Absorbing boundary conditions for the numerical simulation of waves, Math. Comput. 31 (1977) 629.

[9] X. Antoine, A. Arnold, C. Besse, M. Ehrhardt and A. Schädle, A Review of artificial boundary conditions for the Schrödinger equation, Commun. Comput. Phys. 4(4) (2008) 729.

[10] X. Antoine, E. Lorin and Q. Tang, A friendly review of absorbing boundary conditions and perfectly matched layers for classical and relativistic quantum waves equations, Molecular Physics 115(15-16) (2017) 1861.

[11] T. Fevens and H. Jiang, Absorbing boundary conditions for the Schrödinger equation, SIAM J. Sci. Comput. 21(1) (1999) 255.

[12] X. Antoine, C. Besse and P. Klein, Absorbing boundary conditions for the one-dimensional Schrödinger equation with an exterior repulsive potential, J. Comp. Phys. 228(2) (2009) 312.

[13] X. Antoine and C. Besse, Unconditionally stable discretization schemes of non-reflecting boundary conditions for the one-dimensional Schrödinger equation, J. Comput. Phys. 188 (2003) 157.

[14] X. Wu and J. Zhang, High-order local absorbing boundary conditions for heat equation in unbounded domain, J. Comput. Math. 29 (2011) 74.

[15] A. Arnold, M. Ehrhardt and I. Sofronov, Discrete transparent boundary conditions for the Schrödinger equation: Fast calculation, approximation, and stability, Commun. Math. Sci. 1(3) (2003) 501.

[16] L. Greengard and P. Lin, On the numerical solution of the heat equation in unbounded domains (Part I), Tech. Note 98002, Courant Mathematics and Computing Laboratory. New York University, (1998).

[17] X. Wu and Z. Sun, Convergence of difference scheme for heat equation in unbounded domains using artificial boundary conditions, Appl. Numer. Math. 50 (2004) 261.

[18] V. Baskakov and A. Popov, Implementation of transparent boundaries for numerical solution of the Schrödinger equation, Wave Motion. 14 (1991) 123 .

[19] H. Han and Z. Huang, Exact and approximating boundary conditions for the parabolic problems on unbounded domains, Comput. Math. Appl. 44 (2002) 655.

[20] H. Han and Z. Huang, Exact artificial boundary conditions for Schrödinger equation in $\mathbb{R}^{2}$, Comm. Math. Sci. 2(1) (2004) 79.

[21] A. Arnold, M. Ehrhardt, M. Schulte and I. Sofronov, Discrete transparent boundary conditions for the Schrödinger equation on circular domains, Commun. Math. Sci. 10(3) (2012) 889.

[22] H. Li, X. Wu and J. Zhang, Local artificial boundary conditions for Schrödinger and heat equations by using high-order azimuth derivatives on circular artificial boundary, Comp. Phys. Commun. 185 (2014) 1606.

[23] X. Antoine, C. Besse and V. Mouysset, Numerical schemes for the simulation of the two-dimensional Schrödinger equation using nonreflecting boundary conditions, Math. Comput. 73 (2004) 1779.

[24] X. Antoine, C. Besse and P. Klein, Absorbing boundary conditions for general nonlinear Schrödinger equations, SIAM J. Sci. Comput. 33(2) (2011) 1008 .

[25] X. Antoine, C. Besse and P. Klein, Absorbing boundary conditions for the two-dimensional Schrödinger equation with an exterior potential part I: construction and a priori estimates, M3AS, 22 (10) (2012).

[26] X. Antoine, C. Besse and P. Klein, Absorbing boundary conditions for the two-dimensional Schrödinger equation with an exterior potential. Part II: discretization and numerical results, Numer. Math. 228(2) (2013) 312. 
[27] R. Higdon, Absorbing boundary conditions for difference approximations to the multi-dimensional wave equation, Math. Comput. 47 (1986) 437.

[28] G. Pang and S. Tang, Time history kernel functions for square lattice, Comput. Mech. 48 (2011) 699.

[29] S. Katsura and S. Inawashiro, Lattice Green's functions for the rectangular and the square lattices at arbitrary points, J. Math. Phys. 12 (1971) 1622

[30] X. Antoine, W. Bao and C. Besse, Computational methods for the dynamics of the nonlinear Schrödinger/Gross-Pitaevskii equations, (A Feature Article) Comp. Phys. Commun. 184 (12), (2013), 2621. 